\title{
Evaluación nematicida del aceite esencial de Tagetes zypaquirensis en el manejo del nematodo Meloidogyne spp.
}

\author{
Nematicide evaluation of the essential oil from Tagetes zypaquirensis in the control of the nematode \\ Meloidogyne spp.
}

\section{David Eduardo Álvarez S. ${ }^{1}$; Jennifer Aleida Botina J. ${ }^{2}$; Abner Jarminton Ortiz C. ${ }^{2}$; Lorena Lisbetd Botina . $^{3}$}

1. Ingeniero Agrónomo M.Sc. Investigador Grupo GRISAV, Universidad de Nariño. Pasto, Colombia, daealvarezsa@unal.edu.co.

2. Ingeniero Agroforestal, Universidad de Nariño. Pasto, Colombia, jenniferossy@yahoo.es.

3. Ingeniera Agrónoma, Universidad de Nariño. Pasto, Colombia, lisbetdlorena@gmail.com.

Citar: ÁLVAREZ, D.; BOTINA, J.; ORTIZ, A.; BOTINA; L. 2016. Evaluación nematicida del aceite esencial de Tagetes zypaquirensis en el manejo del nematodo Meloidogyne spp. Rev. Cienc. Agr. 33(1): 22-33.

Fecha recibido: Junio 04 de $2015 . \quad$ Fecha de aceptación: Noviembre 26 de 2015.

\section{RESUMEN}

El lulo (Solanum quitoense Lam.) es un frutal andino de gran importancia económica en Colombia, sin embargo, las áreas sembradas y rendimientos han sufrido una evidente reducción debido al ataque de patógenos como el nematodo Meloidogyne spp., que ha ocasionado pérdidas de hasta 50\%. En la naturaleza existen diferentes recursos vegetales con propiedades nematicidas, destacándose al género Tagetes. El objetivo de esta investigación fue evaluar la actividad nematicida del aceite esencial de Tagetes zypaquirensis sobre Meloidogyne spp. Bajo condiciones de invernadero se evaluaron cuatro concentraciones del aceite esencial (100, $200,400,800 \mathrm{mg} / \mathrm{kg}$ de suelo) las cuales fueron aplicadas a un suelo con juveniles de segundo estadio del nematodo $\pm 400 \mathrm{~J} 2 / 100 \mathrm{~g}$. Además, se establecieron tres comparadores: un tratamiento sin aplicación de aceite esencial, un suelo sin nematodo y un suelo tratado con i.a carbofuran (33,2\%) bajo una dosis de 2cc/L. Para cada tratamiento, las variables a evaluar fueron: severidad, altura de planta, peso fresco y seco al aire, peso fresco radical, número de huevos del nematodo/100g raíz y número de J2/100g de suelo. Los componentes mayoritarios del aceite esencial de T. zypaquirensis fueron dihidrotagetona y $E$-tagetona con una proporción relativa de 42,2 y 22,9\%, respectivamente. Los resultados indicaron, que la concentración $800 \mathrm{mg} / \mathrm{kg}$ de aceite 
esencial presentó la misma acción nematicida que el suelo tratado con carbofuran al reducir las poblaciones de Meloidogyne spp. y presentar valores similares en las variables fitométricas. Se concluye que el aceite esencial T. zypaquirensis puede ser una alternativa para el manejo de la enfermedad del nudo radical.

Palabras clave: nudo radical, aceite esencial, dihidrotagetona, E-tagetona, Solanum quitoense Lam.

\begin{abstract}
Lulo (Solanum quitoense Lam.), an Andean fruit, is economically important in Colombia, however, cultivated areas as well as yields, have suffered a considerable reduction by the attack of pathogens such as the rootknot nematode Meloidogyne spp. with losses near to 50\%. In nature, there are different plant with nematicides properties which could diminish the effects of this nematode, such as the Tagetes genre. The objective of this research was to evaluate the nematicidal activity of the essential oil from Tagetes szypaquirensis on Meloidogyne spp. Four concentrations of essential $(100,200,400,800 \mathrm{mg} / \mathrm{kg}$ of soil) were evaluated under greenhouse conditions. These doses were applied to nematodes in the second instar approximately \pm 400 J2/100g of soil. Moreover, three treatments were established: the first with no application of essential oil, the second without nematode, and the third with soil treated with carbofuran (33.2\%) at dose of 2cc/L. For each treatment the variables evaluated were: level of severity, plant height, fresh and dry weight, fresh root weight, number of nematode eggs $/ 100 \mathrm{~g}$ roots and number of J2/100g of soil. The main components of the essential oil from T. zypaquirensis were dihydrotagetone and E-tagetone with a composition of $42.2 \%$ and $22.9 \%$ respectively. The results indicated that a concentration $800 \mathrm{mg} / \mathrm{kg}$ of essential oil had the same nematicidal action than soil treated with carbofuran, same population constrains and phitometric variables. It was concluded that T. zypaquirensis essential oil can be an alternative for managing root knot disease.
\end{abstract}

Key works: root-knot, essential oil, dihydrotagetone, E-tagetone, Solanum quitoense Lam.

\section{INTRODUCCIÓN}

El lulo Solanum quitoense Lam., es un frutal andino de gran importancia económica debido a las características nutricionales y agroindustriales que lo hacen apetecible en los mercados nacionales e internacionales, siendo los principales importadores la Unión Europea, Japón, Corea y Estados Unidos. Colombia exportó un promedio de 120 toneladas durante el 2012 (MADR, 2012). El área cultivada de este frutal en Colombia es de 7.327ha, con una producción promedio de 68.748t, sobresaliendo el departamento del Huila como el mayor productor con 13.669t, le siguen los departamentos del Valle del Cauca, Antioquia, Tolima, Santander y Nariño. Este último participa a nivel nacional con 434ha y una producción de 2,108t siendo un frutal representativo de la región norte del departamento (MADR, 2013).
Sin embargo, en Nariño la tendencia de área sembrada y rendimiento de lulo, ha sufrido una evidente reducción desde el año 2005, pasando de 526ha cosechadas a 434ha en el 2013, reportando una tasa de crecimiento promedio anual negativa de -5,25\% (MADR, 2013). Esta reducción se debe entre muchos factores a la presencia del nematodo Meloidogyne spp. causante del problema fitosanitario "nudo radical del lulo" el cual afecta los municipios de San Pedro de Cartago, El Tambo, Ancuya, Buesaco, La Unión, San Lorenzo y Consacá (Chaves et al., 2002; Gelpud et al., 2011; Alvarez et al., 2016).

Los nematodos de agallas de raíces entre los cuales se encuentra Meloidogyne spp. son los más importantes a nivel mundial, tanto por su amplia distribución como por el elevado número de familias y especies de plantas que afecta (Tamayo et al., 2003; 
Gelpud et al., 2011). En el cultivo de lulo provocan hinchazones o agallas en la raíz que disminuyen significativamente los rendimientos y la vida de la planta afectada, pasando de una vida útil de cinco a dos años (Tamayo et al., 2003). Esto se debe posiblemente a la disminución de la absorción de agua y nutrientes y por el ingreso de microorganismos causantes de enfermedades radiculares (Tamayo et al., 2003; Castro et al., 2011).

La estrategia de control utilizada por el agricultor frente a la enfermedad normalmente se basa en el uso de plaguicidas, los cuales incrementan los costos de producción y afectan la dinámica del suelo al ser utilizadas moléculas de los grupos de los organofosforados, carbamatos y algunos productos fumigantes de alta residualidad. La aplicación de estos productos catalogados como nematicidas, causan fuertes problemas de contaminación ambiental (Tamayo et al., 2003; Murcia y Stashenko, 2008; Alvarez et al., 2016).

Ante esta problemática se considera necesario evaluar alternativas menos contaminantes como el uso de estrategias biosostenibles (Múnera, 2002), dentro de las cuales, se encuentran el empleo de aceites esenciales (A.E.) extraídos de plantas aromáticas, que son una opción importante para controlar insectos (Serrato et al., 2007; Díaz et al., 2012), hongos (Talamini y Stadnik, 2004; Serrato et al., 2007) y nematodos (Taylor y Sasser, 1983; Ploeg, 2000; Rodríguez et al., 2001; Pérez et al., 2003; Riga, 2005; Serrato et al., 2007). Estos compuestos naturales presentan características biodegradables y no persistentes (Kimpinski et al., 2000). Entre las especies promisorias se destaca la utilización de plantas del género Tagetes (Asteraceae), el cual ha sido reconocido por producir compuestos nematicidas como $E$-tagetona, cis-ocimeno, dihidrotagetona, entre otros (Ploeg, 2000; Kimpinski et al., 2000).

La especie T. zypaquirensis, es un arbusto perenne que se encuentra en las cordilleras Colombianas, conocida por los múltiples beneficios etnobotánicos. También es considerada una planta con características plaguicidas por sus compuestos alelopáticos, los cuales pueden ser liberados por volatilización, exudados radiculares, lixiviación desde las plantas y por descomposición de residuos (Tsay y Lin, 2004). Los compuestos que emana son generalmente metabolitos secundarios que actúan como defensa química ante enfermedades y parásitos (Pérez et al., 2003; Tsay y Lin, 2004). Dentro de la composición química que posee la especie de T. zypaquirensis se encuentran los grupos monoterpenos, sesquiterpenos, esteres de etilo y ácidos grasos, compuesto tóxico para algunos nematodos (Díaz et al., 2012).

Por lo anterior, el objetivo de esta investigación fue evaluar el efecto nematicida del aceite esencial de T. zypaquirensis en el manejo del nematodo Meloidogyne spp. causante del nudo radical del lulo, con el fin de proponer un manejo alternativo a este problema fitosanitario.

\section{MATERIALES Y MÉTODOS}

Localización. La investigación se desarrolló en el invernadero y planta piloto de la Universidad de Nariño, localizados en el municipio de Pasto a $2.525 \mathrm{msnm}$ y una temperatura que oscila entre 13 a $18^{\circ} \mathrm{C}$.

Material vegetal. Se identificaron arbustos de $T$. zypaquirensis, en praderas naturales del municipio de Pasto, corregimiento de Cabrera (112' $8^{\prime \prime} \mathrm{LN}$ y $\left.77^{\circ} 12^{\prime} 15^{\prime \prime} \mathrm{LO}\right)$. Hojas e inflorescencias, fueron colectadas de forma masiva y llevadas a la Planta Piloto de la Universidad de Nariño para la extracción de su aceite esencial.

Extracción del aceite esencial. Se utilizó la técnica de hidrodestilación simple modificada de Quintero y Gonzales (2004), mediante montaje tipo Clevenger se hizo pasar agua a una temperatura de 100 a $105^{\circ} \mathrm{C}$ durante $2 \mathrm{~h}$ a través de un matraz balón de $1 \mathrm{~L}$ que contenía $500 \mathrm{~g}$ de material vegetal de T. zypaquirensis. La muestra agua-aceite fue condensada y luego separada empleando un 
embudo de decantación. El aceite obtenido se secó con sulfato de sodio anhidro y se almacenó a $8^{\circ} \mathrm{C}$ hasta su utilización.

Perfil cromatográfico del aceite esencial. El análisis químico del aceite de T. zypaquirensis, se realizó mediante cromatografía de gas vinculado a espectrometría de masas (CG-MS) siguiendo la metodología con modificaciones de Barbosa et al. (2006), utilizando un equipo Shimadzu QP-20105 CGMS equipado con un inyector split/splitless a temperatura de $250^{\circ} \mathrm{C}$ (relación split $1: 100$ ) y un detector de ionización en llama (FID) $\left(280^{\circ} \mathrm{C}\right)$. Los datos cromatográficos, fueron adquiridos y procesados con el programa Shimadzu Class VP 4.3. Para la separación del aceite esencial, se utilizó una columna apolar DB-5 (J\&W) de $30 \mathrm{~m}$ x 0,25mm D.I. y $0,25 \mu \mathrm{m}$ de una fase estacionaria de $5 \%$ fenil-polimetilsiloxano. La temperatura del horno fue programada de $40^{\circ} \mathrm{C}(5 \mathrm{~min})$ hasta $250^{\circ} \mathrm{C}$ a $5^{\circ} \mathrm{Cmin}^{-1}$. El gas de arrastre fue Helio (99,99\%, Aga-Fano S.A) a flujo de $1 \mathrm{ml} \cdot \mathrm{min}^{-1}$. El volumen inyectado del A.E. fue de 1,0 $\mu \mathrm{L}$. Los espectros de masas se obtuvieron en un intervalo de masas de 38 a $450 \mathrm{~m} / \mathrm{z}$.

La identificación de los compuestos, se realizó utilizando los índices de retención de Kovats, así como por comparación de los espectros de masas obtenidos con aquellos de la librería de espectros Wiley. La cuantificación se realizó mediante el cálculo del porcentaje relativo de áreas de cada compuesto.

Evaluación de la actividad nematicida del aceite esencial. El aceite esencial de T. zypaquirensis fue diluido en una solución de agua estéril que contenía una relación de etanol (grado analítico) y Tween-20 al 5\% (v/v). Esta solución final fue utilizada para calcular las diferentes dosis del ensayo en invernadero.

Para determinar el efecto del aceite esencial sobre el nematodo causante del nudo radical, se utilizó la metodología descrita por Oka et al. (2000) disponiendo en bolsas plásticas $1.500 \mathrm{~g}$ de suelo inoculado con el nematodo de Meloidogyne spp. $\pm 400 \mathrm{~J} 2$ por gramos de suelo, obtenidos previamente por el método de centrifugación y flotación en sacaro- sa (Castro et al., 2011). Seguidamente, el aceite se aplicó en el suelo bajo una relación de 100, 200, 400 y $800 \mathrm{mg} / \mathrm{Kg}$. Adicionalmente, se establecieron tres comparadores: un testigo químico con aplicación de nematicida comercial carbofuran (i.a. $33,2 \%$ ) bajo una dosis de $100 \mathrm{cc} / \mathrm{L}$, un tratamiento con suelo sin presencia del nematodo esterilizado en autoclave (15 psi, 15 minutos) y suelo infestado con el nematodo sin la aplicación de aceite esencial. Los tratamientos se describen en la Tabla 1.

Cinco días después de la disposición de los tratamientos, una planta de lulo de cuatro semanas de edad, fue trasplantada a cada bolsa plástica.

Tabla 1. Descripción de tratamientos, concentración de aceite esencial e inoculación del nematodo a nivel de invernadero.

\begin{tabular}{ccc}
\hline Tratamiento & Característica & $\begin{array}{c}\text { PN }_{\mathbf{i}}(\mathrm{J} 2 / \mathbf{1 0 0 g} \\
\text { suelo)* }\end{array}$ \\
\hline T1 & A.E. $100 \mathrm{mg} / \mathrm{Kg}$ & \pm 400 \\
T2 & A.E. $200 \mathrm{mg} / \mathrm{Kg}$ & \pm 400 \\
T3 & A.E $400 \mathrm{mg} / \mathrm{Kg}$ & \pm 400 \\
T4 & A.E. $800 \mathrm{mg} / \mathrm{Kg}$ & \pm 400 \\
T5 & Carbofuran & \pm 400 \\
T6** & Agua destilada & $\pm 0,0$ \\
T7*** & Agua destilada & \pm 400 \\
\hline
\end{tabular}

* $\mathrm{PN}_{\mathrm{i}}=$ Población inicial de J2 de Meloidogyne spp. inoculada al suelo; ${ }^{* *}$ T6 = Testigo 1.; ${ }^{* * * T 7}=$ Testigo 2.

Diseño experimental. Se utilizó el Diseño Irrestrictamente al Azar (DIA) con siete tratamientos y diez repeticiones para un total de 70 unidades experimentales. Los valores de cada variable se tomaron de cada unidad experimental constituida por una planta de lulo en cada tratamiento (Tabla 1).

Variables evaluadas. Pasados 110 días del establecimiento del ensayo, en las unidades experimentales se evaluaron las siguientes variables, mediante una prueba destructiva de las plantas de lulo. 
Incidencia. Se calculó el porcentaje de plántulas infestadas por el nematodo en las 10 plantas de cada tratamiento, utilizando la fórmula:

Infección (\%) = (No Plántulas infestadas $/$ Total de plántulas) x 100 .

Severidad. Se evaluó con base en el índice de nudosidad, determinando el número de agallas y/o masas de huevos por planta, utilizando la escala de infección radical propuesta por Taylor y Sasser (1983), representada en la Figura 1.

Altura de planta (AP). Se midió la altura de cada una de las plantas desde la base hasta el ápice del tallo, llevando un registro cada 15 días, a partir del momento del trasplante hasta completar la investigación (110 días).

Peso fresco de raíz (PFR). Se separó con la ayuda de un bisturíla raíz y el tallo de cada planta para su posterior pesaje en balanza analítica $(\mathrm{g})$ tomando el total de plantas del tratamiento.

Peso fresco y seco aéreo (PFA). Se pesaron las hojas y tallos de cada planta, con la ayuda de una balanza analítica se determinó el peso fresco (g). Seguidamente, el material fue empacado en bolsas de papel rotuladas llevándose al horno a $65^{\circ} \mathrm{C}$ hasta obtener un peso constante, determinando el peso seco aéreo (g).

Conteo de J2 de Meloidogyne spp. Se utilizó el método de extracción conteo con solución azucarada descrito por Caveness y Jensen (1955). En la primera fase, se tomaron muestras de $100 \mathrm{~g}$ de suelo, cada muestra fue lavada a presión con
5L de agua en un balde de 10L de capacidad. El suelo en suspensión, se dejó sedimentar durante 20 segundos. Al cabo de este periodo se separó el sobrenadante a través de un juego de cribas de mallas de 60, 125 y 325micrones. En la segunda fase, el residuo de suelo retenido en la criba de 325 micrones fue dispuesto en un tubo de centrifugación de $50 \mathrm{~mL}$ para iniciar un ciclo de centrifugado a 1.800rpm durante 3 minutos. El líquido sobrenadante, fue descartado y posteriormente fue sustituido por una solución azucarada con una densidad específica de 1,17 (471g/L de solución); la muestra fue centrifugada una segunda vez a $1.800 \mathrm{rpm}$ por 3 minutos. El sobrenadante, fue vertido a través de una criba de 325 micrones y lavado con abundante agua. Posteriormente, la suspensión de nematodos fue pasada con ayuda de un gotero a la rejilla de conteo para la determinación de J2 usando un microscopio (Nikon SMZ645).

Conteo de huevos de Meloidogyne spp. Se tomaron al azar cuatro raíces por tratamiento. La extracción de huevos para su conteo se realizó macerando $100 \mathrm{~g}$ de raíces en $500 \mathrm{~mL}$ de agua destilada utilizando una licuadora durante 15 segundos. La solución obtenida se vertió en cribas de 60 y 325 micrones. El número de huevos por sistema radical, fue determinado utilizando una rejilla de conteo para nematodos con la ayuda de un estereoscópico a 60X (Elyousr et al., 2010).

Análisis estadístico. Las variables evaluadas se sometieron al Análisis de Varianza (ANDEVA). Encontradas diferencias significativas se realizó una prueba de comparación de medias DMS al $5 \%$ de confianza mediante el paquete estadístico Infostat (Di Rienzo et al., 2013).

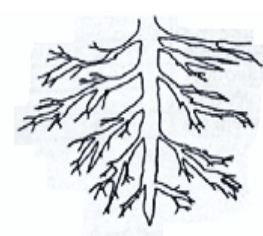

$0 \%$
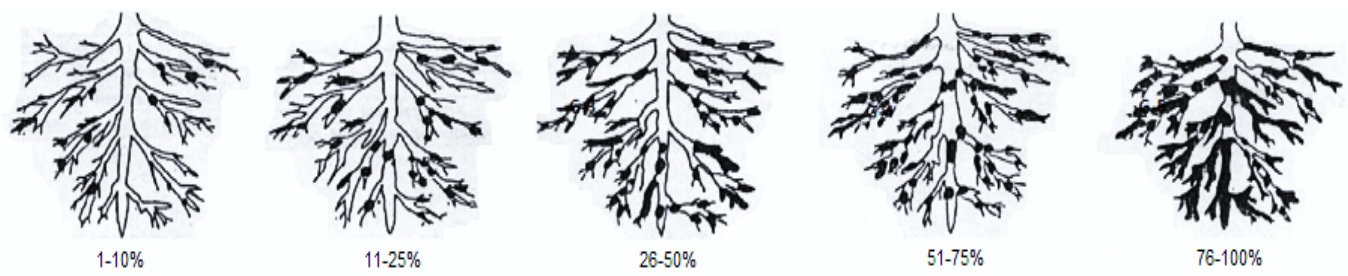

Figura 1. Escala de infección radical causado por Meloidogyne spp. 


\section{RESULTADOS Y DISCUSIÓN}

Perfil cromatográfico del aceite esencial. Se identificaron 10 compuestos principales, que acorde con su patrón de fragmentación, aparecieron con tiempos de retención de Kovats en la secuencia que se indica en la Tabla 2. La dihidrotagetona se reporta como el compuesto mayoritario con una cantidad relativa de $42,2 \%$, seguido de la $E$-tagetona con $22,9 \%$, por último la trans-ocimenona con 20,8\%. Los resultados obtenidos, son similares en estudios realizados por Escobar et al. (2009) quienes identificaron los componentes principales de T. zypaquirensis de la región de Cundinamarca, con una cantidad relativa del $42,0 \%$ de dihidrotagetona, E-tagetona con 5,8 y $12,3 \%$ de trans-ocimenona. La diferencia de las proporciones encontradas de los compuestos, pueden explicarse por las condiciones climáticas, estado de crecimiento del arbusto, así como también del método y las condiciones de extracción del aceite esencial (Serrato et al., 2007). En estudios realizados por Stefanazzi et al. (2006), Serrato et al. (2007) y Díaz et al. (2012) señalan que la E-tagetona se encuentra presente en las especies de T. terniflora, T. lacera y T. zypaquirensis en cantidades relativas entre el 10,7 y el $27 \%$.

Tabla 2. Composición química del aceite esencial de T. zypaquirensis.

\begin{tabular}{cccl}
\hline No. & TR $^{*}$ & CR (\%)** & Identificación \\
\hline 1 & 12,195 & 2,2 & Cis-ocimeno \\
2 & 12,658 & 42,2 & Dihidrotagetona \\
3 & 13,293 & 1,3 & No identificado \\
4 & 14,257 & 0,7 & Z-tagetona \\
5 & 14,846 & 22,9 & E-tagetona \\
6 & 15,785 & 5,6 & Cis-ocimenona \\
7 & 15,995 & 20,8 & Trans-ocimenona \\
8 & 18,342 & 0,4 & $\alpha$-farneseno \\
9 & 19,884 & 1,4 & Isocariofileno \\
10 & 20,982 & 0,5 & Espatulenol \\
\hline
\end{tabular}

${ }^{*} \mathrm{TR}=$ Tiempo de retención $(\mathrm{min}),{ }^{* *} \mathrm{CR}=$ Cantidad relativa.
En cuanto a la trans-ocimenona según Stefanazzi et al. (2006) y Escobar et al. (2009) reportan que en especies como T. terniflora, T. caracasana y T. zypaquirensis sus cantidades relativas corresponden a 17,5, 1,8 y 12,3\%, respectivamente. En general, para Adekunle et al. (2007) el metabolito dihidrotagetona constituye uno de los principales compuestos bioactivos del género Tagetes.

Incidencia. Los resultados obtenidos 110 días después del trasplante de las plántulas de lulo a las unidades experimentales, indicaron que el 100\% de las plantas en cada tratamiento presentaron afecciones del nematodo (Figura 2). Sin embargo, el tratamiento T4 (A.E. $800 \mathrm{mg} / \mathrm{kg}$ ) al igual que T5 (i.a. carbofuran) ejercieron una mayor regulación nematicida al encontrarse un menor número de agallas radicales relacionado al valor de severidad de la enfermedad. Hay que destacar, que el cultivo de lulo presenta un alto grado de susceptibilidad al ataque del nematodo Meloidogyne como lo manifiesta Taylor y Sasser (1983), Tamayo et al. (2003) y Gelpud et al. (2011).

Resultados similares fueron reportados por Parada y Guzmán (1997) quienes evaluaron extractos acuosos de ajo (Allium satium L.), papaya (Carica papaya L.), pasto barrenillo (Cynodon dactylon L.) y marygold (Tagetes sp.) con el propósito de determinar sus efectos nematicidas sobre Meloidogyne spp. en frijol (P. vulgaris), obteniendo valores del $100 \%$ de incidencia 60 días después de la inoculación del nematodo, no obstante, para estos autores la acción nematicida del extracto fue determinada por el grado de severidad de las plantas de frijol comparadas con el testigo absoluto, utilizándose este procedimiento en esta investigación.

Severidad. Se presentó variación en los grados de severidad en los diferentes tratamientos (Figura 2 y Tabla 3). Dentro de los tratamientos con aplicación de A.E., la concentración de aceite esencial $100 \mathrm{mg} / \mathrm{kg}$ se presentó el mayor porcentaje de afección con valores porcentuales entre el 51 y 75\% de la escala de afección radical; los trata- 


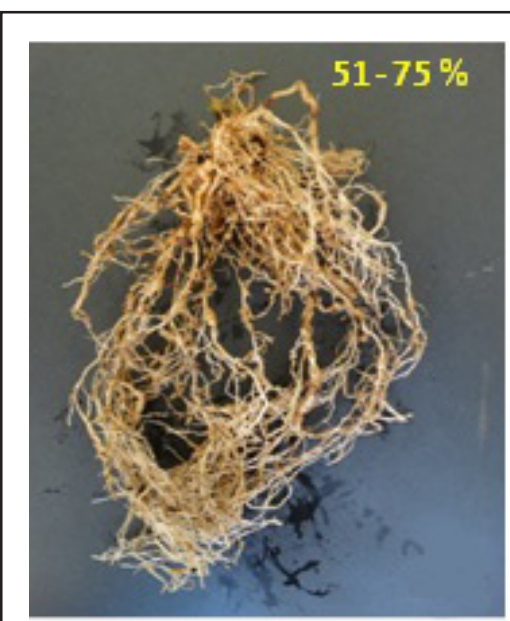

T1

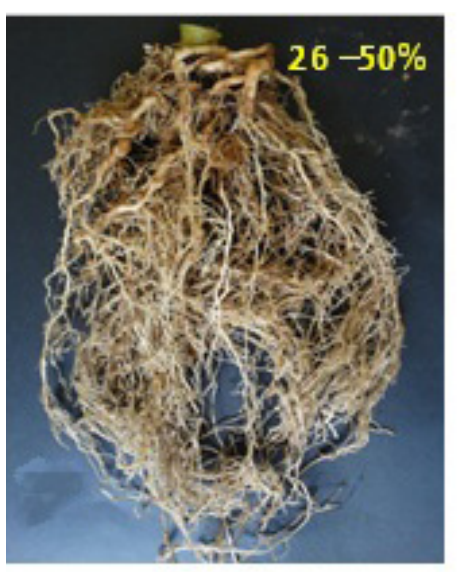

T4

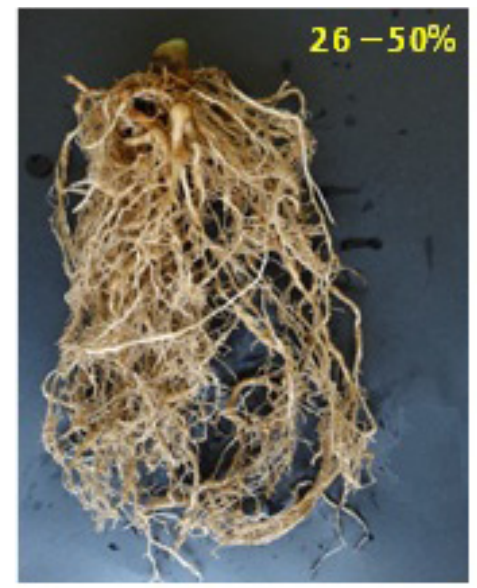

$\mathrm{T} 2$

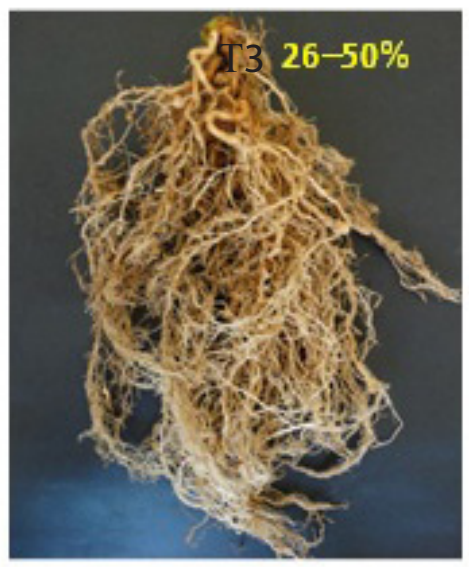

T5

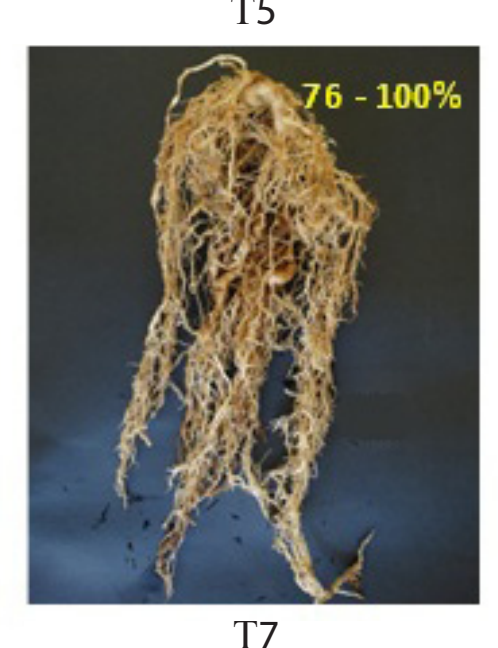

T7

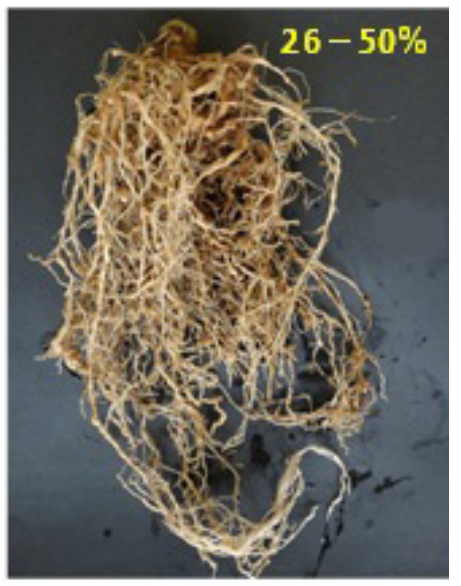

T3

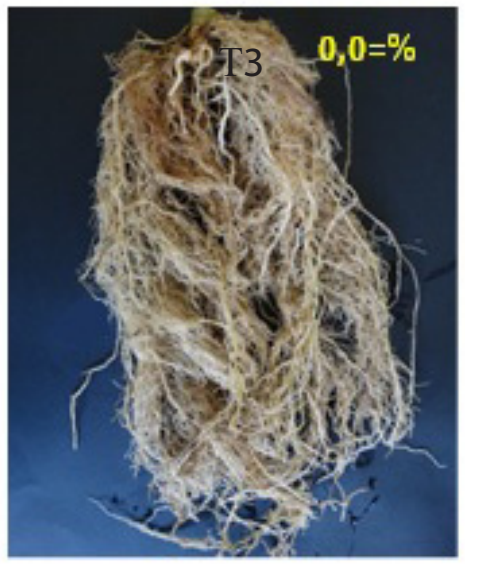

T6

Figura 2. Reacción de severidad de la enfermedad del nudo radical del lulo a la aplicación del A.E. de T. zypaquirensis. T1 = A.E. $100 \mathrm{mg} / \mathrm{Kg}$, T2 = A.E. $200 \mathrm{mg} / \mathrm{Kg}, \mathrm{T} 3=$ A.E. $400 \mathrm{mg} / \mathrm{Kg}$, T4 = A.E. $800 \mathrm{mg} / \mathrm{Kg}$, T5 = i.a carbofuran (33,2\%), T6 = Agua destilada+ 0,0 J2 y T7 = Agua destilada+ $400 \mathrm{~J} 2$. 
mientos T2, T3 y T4 así como el tratamiento T5 (i.a. carbofuran) presentaron valores entre el 26 y $50 \%$ de severidad observándose nudosidades alargadas, aunque, el sistema radical no se afectó totalmente. El tratamiento $\mathrm{T} 6( \pm 0,0 \mathrm{~J} 2 / 100 \mathrm{~g})$ expresó un desarrollo radicular normal y un valor de $0 \%$ de severidad de la enfermedad. En cuanto al tratamiento T7 ( $₫ 400 \mathrm{~J} 2 / 100 \mathrm{~g})$ como comparador absoluto (Testigo 2) presentó el máximo valor de severidad en la investigación con un promedio del 76 al 100\% de afección radical (Tabla 3), donde la nutrición de la planta es interrumpida existiendo pudrición del tejido afectado y comprometiéndose los procesos de absorción de nutrientes en las plantas evaluadas (Taylor y Sasser, 1983).

En estudios realizados en piña (A. comosus) por Rodríguez et al. (2001) evaluado extractos botánicos, entre ellos el de Tagetes sp. frente al nematodo Meloidogyne spp. concluyen que la aplicación del extracto acuoso al suelo disminuyó el índice de agallamiento demostrando la acción biocida del género Tagetes. Estos resultados respaldan los valores obtenidos en esta investigación, referente a la disminución de los índices de severidad de la enfermedad.

Díaz et al. (2012) indican que las especies Tagetes patula, Tagetes erecta y Tagetes filifolia son un recurso de control eficaz frente a una amplia gama de nematodos al presentar en su composición metabolitos como la tagetona, que en conjunto con otros compuestos como la dihidrotagetona, crisantenona, alilanisol y el canfeno presentan actividad biocida. Al respecto, se ha planteado la hipótesis, de que algunos aceites esenciales interfieren en el sistema nervioso central de los nematodos.

Peso fresco radical (PFR). Se separaron cuatro grupos estadísticos como se describen en la Tabla 3. El tratamiento T7 (Testigo 2) presentó el valor de PFR más bajo en la investigación con 20,37g observándose escasez de raíces y bajo vigor. El siguiente grupo lo conformaron los tratamientos T1,
T2, T3 y T5 con un valor promedio de 29,11g. El tratamiento T4 en el cual se aplicó la mayor dosis del aceite esencial de T. zipaquirensis presentó diferencias estadísticas con las demás concentraciones y superó también a la aplicación de carbofuran, el cual reportó 33,07g. Finalmente, el comparador absoluto (T6) en el cual no se inoculó el nematodo el valor de PFR fue superior al promedio del ensayo con 51,01g (Tabla 3).

Hay que tener en cuenta que la severidad de la enfermedad presentada en los tratamientos con la aplicación de aceite esencial fue menor al 50\%, Taylor y Sasser (1983) y El-Sherif et al. (2007) señalan que las raíces infestadas con Meloidogyne spp. son más cortas que las raíces sanas, tienen menos raíces laterales y menos pelos radicales reduciendo el peso radical, lo cual concuerda con los resultados de ésta investigación.

En estudios realizados por Lehman (1979) concluyeron que los extractos acuosos de T. patula, T. erecta y T. minuta afectaron las poblaciones del nematodo de la papa Pratylenchus penetrans obteniendo reducciones del 63\%, asociado al mayor peso del sistema radical de la planta.

Peso fresco aéreo (PFA) y peso seco aéreo (PSA). El tratamiento T6 (Testigo 1) obtuvo el mayor valor de peso fresco aéreo en la investigación con 114,89g, seguidamente T5 (carbofuran) con 91,68g. En relación a los tratamientos con aplicación del aceite esencial T1 a T4 se estableció un valor promedio de 74,35g presentando diferencias significativas respecto al testigo absoluto con presencia del nematodo (T7) el cual reporto el peso más bajo como se esperaba. En cuanto al peso seco aéreo (PSA) los valores respaldan fielmente la información obtenida en PFA como se observa en la Figura 3. Se resalta el valor de las plantas con aplicación de A.E $800 \mathrm{mg} / \mathrm{Kg}$ de suelo (T4) las cuales presentaron un promedio de $8,62 \mathrm{~g}$ siendo solamente superada por el Testigo sin inoculación del nematodo. 


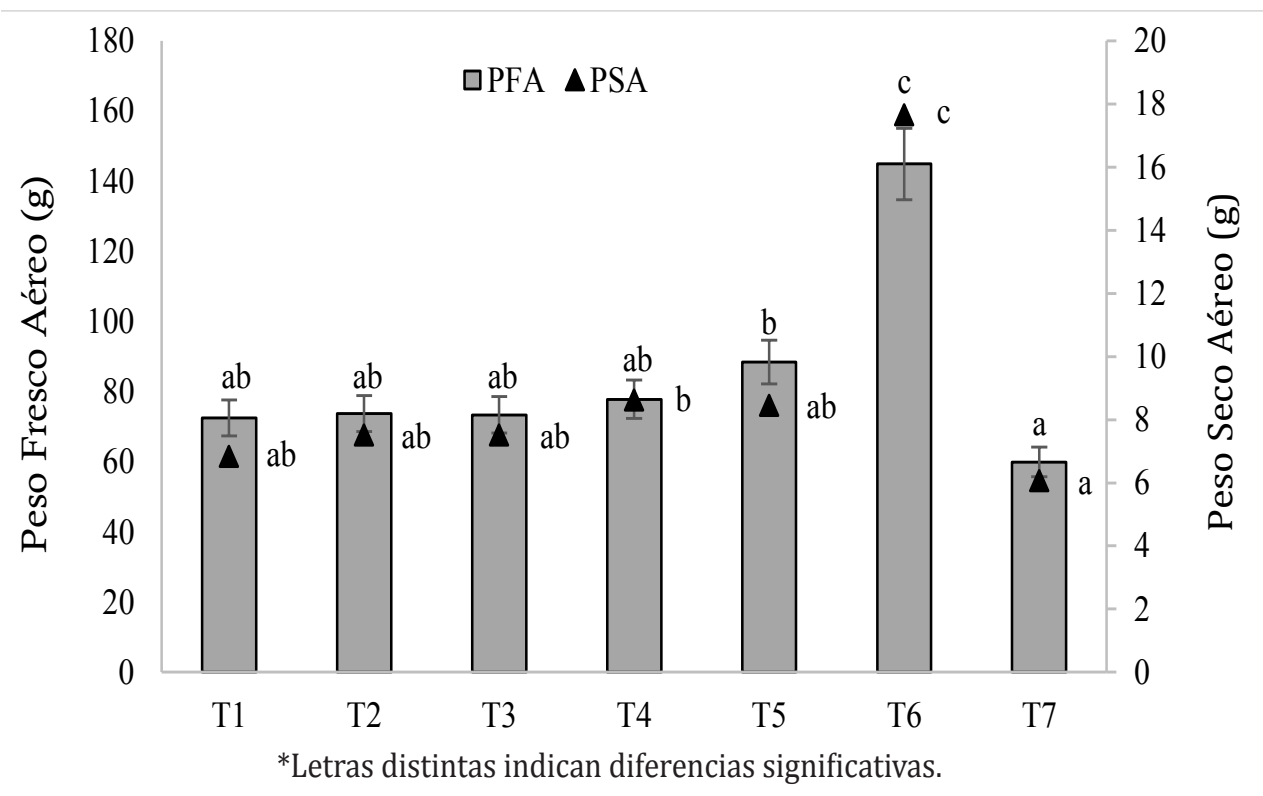

Figura 3. Peso fresco aéreo (PFA) y peso seco aéreo (PSA), 110 dias después del transplante de las plantas de lulo.

Según Múnera (2002) y El-Sherif et al. (2007) los síntomas del nematodo Meloidogyne spp. sobre la zona aérea de la planta afectada, se expresan en la inhibición del crecimiento meristemático, disminución del crecimiento foliar, deficiencias nutricionales en forma de clorosis del follaje y senescencia de hojas; lo anterior se relaciona a la interferencia causada por el nematodo en la producción y translocación de sustancias provenientes de las raíces, los elementos vasculares en los nódulos se rompen y deforman interrumpiendo mecánicamente el flujo normal de agua y nutrientes, Taylor y Sasser (1983), Castro et al. (2011), Tamayo et al. (2003) y Gelpud et al. (2011) señalan que los anteriores síntomas están directamente relacionados con el nivel poblacional del nematodo.

Altura de planta (AP). Los resultados obtenidos indicaron que los tratamientos T1 y T7 presentaron las plantas de menor altura con medidas de 29,44 y $26,88 \mathrm{~cm}$, respectivamente, siendo éstos estadísticamente diferentes a los demás tratamientos (T2 a T6). Ploeg (2000), Kumar y Khanna
(2006) afirman que la reducción en la altura de las plantas se debe a una alta densidad del patógeno que impide la adecuada expansión de las raíces interrumpiendo los procesos fisiológicos normales de la planta.

Esta variable presentó un valor estadísticamente superior con la aplicación de la dosis de A.E. de T. zypaquirensis $800 \mathrm{mg} / \mathrm{kg}$ frente al Testigo 1 (T6), indicando alta eficiencia en el manejo de la enfermedad del nudo radical. Adegbite y Adesiyan (2006) señalan que a un mayor tiempo de exposición o dosis de los extractos de T. patula afectó en mayor proporción la capacidad infectiva del nematodo Meloidogyne spp.; sumado a esto, Talamini y Stadnik (2004) describen que en muchos casos, los aceites esenciales presentan compuestos bioactivos capaces de inducir o activar los mecanismos de defensa de la planta y también los llamados bioestimulantes de crecimiento en diferentes especies vegetales, brindando una posible explicación a los resultados obtenidos en esta investigación en donde a mayor dosis de aceite esencial se presentó un mayor crecimiento vegetativo. 
Tabla 3. Aceite esencial de T. zypaquirensis sobre las variables fitométricas e índice de agallamiento en plantas de lulo*.

\begin{tabular}{ccccccc}
\hline Tratamiento & PFA (g) & PSA (g) & PFR (g) & AP (cm) ${ }^{* *}$ & $\mathbf{G}_{\mathbf{f}}$ & \% Nudosidad \\
\hline T1 & $72,50 \mathrm{ab}$ & $6,83 \mathrm{ab}$ & $26,03 \mathrm{ab}$ & $29,44 \mathrm{a}$ & 4,0 & $51-75$ \\
T2 & $73,73 \mathrm{ab}$ & $7,51 \mathrm{ab}$ & $29,06 \mathrm{ab}$ & $32,06 \mathrm{~b}$ & 3,2 & $26-50$ \\
T3 & $73,39 \mathrm{ab}$ & $7,52 \mathrm{ab}$ & $30,07 \mathrm{ab}$ & $30,44 \mathrm{~b}$ & 3,2 & $26-50$ \\
T4 & $77,80 \mathrm{ab}$ & $8,62 \mathrm{~b}$ & $33,07 \mathrm{~b}$ & $34,86 \mathrm{c}$ & 3,0 & $26-50$ \\
T5 & $91,68 \mathrm{~b}$ & $8,46 \mathrm{ab}$ & $27,31 \mathrm{ab}$ & $32,13 \mathrm{c}$ & 3,2 & $26-50$ \\
T6 & $114,89 \mathrm{c}$ & $17,66 \mathrm{c}$ & $51,01 \mathrm{c}$ & $32,06 \mathrm{~b}$ & 0,0 & 0 \\
T7 & $59,94 \mathrm{a}$ & $6,07 \mathrm{a}$ & $20,37 \mathrm{a}$ & $26,88 \mathrm{a}$ & 5,0 & $76-100$ \\
C.V. & $\mathbf{1 5 , 3 1}$ & $\mathbf{1 6 , 5 8}$ & $\mathbf{2 0 , 2 7}$ & $\mathbf{1 2 , 5 9}$ & - & - \\
\hline
\end{tabular}

*Los datos corresponden al promedio de 10 repeticiones** $\mathrm{G}_{\mathrm{f}}=$ Grados de afección; letras distintas indican diferencias significativas.

Conteo de huevos en raíz. Se evidenció la actividad nematicida del aceite esencial de T. zypaquirensis sobre el número de huevos de Meloidogyne spp. ya que en todos los tratamientos evaluados se observó una reducción de estructuras reproductivas (Tabla 4). El análisis estadístico demostró un comportamiento de mayor eficiencia en los tratamientos T4 (A.E. $800 \mathrm{mg} / \mathrm{kg}$ ) así como en el tratamiento T5 (carbofuran) con 2.081,87 y $2.923,67$ huevos/100g raíz. Seguidamente, se encuentra un grupo estadístico conformado por los tratamientos T3, T2 y T1 con 2.832, 3.655 y 3.570 huevos/100g raíz, se prevé una relación inversa entre la adición del aceite esencial y el número de estructuras reproductivas. La mayor cantidad de huevos se presentó comprensiblemente en el tratamiento T7 (Testigo 2) con 4,315 huevos/100g raíz al tener las condiciones favorables para el desarrollo del nematodo.

Estudios realizados por Oka et al. (2000) respaldan los resultados obtenidos en esta investigación al indicar que el aceite esencial de Tagetes sp. disminuyó el número de huevos de Meloidogyne javanica en plantas de tomate bajo invernadero. Los resultados de Chitwood (2002) revelan que los monoterpenos, sesquiterpenos, alcoholes y ésteres de los aceites esenciales pueden afectar las poblaciones de nematodo inviabilizando la eclosión de los huevos, disminuyendo el grado de severidad de la enfermedad, como se pudo determinar en con el aceite de T. zypaquirensis.
Tabla 4. Efectividad del aceite esencial de T. zypaquirensis en el control huevos y J2*.

\begin{tabular}{ccc}
\hline Tratamiento & $\mathbf{M H}_{\mathbf{r}}^{* *}$ & $\mathbf{J}_{\mathrm{s}}{ }^{* * *}$ \\
\hline $\mathrm{T} 1$ & $3.655,40 \mathrm{~d}$ & $357,70 \mathrm{c}$ \\
$\mathrm{T} 2$ & $3.570,47 \mathrm{~d}$ & $316,87 \mathrm{~b}$ \\
$\mathrm{~T} 3$ & $2.832,20 \mathrm{c}$ & $303,80 \mathrm{~b}$ \\
$\mathrm{~T} 4$ & $2.081,87 \mathrm{~b}$ & $254,80 \mathrm{~b}$ \\
$\mathrm{~T} 5$ & $2.923,67 \mathrm{c}$ & $251,53 \mathrm{~b}$ \\
$\mathrm{~T} 6$ & $0,00011 \mathrm{a}$ & $0,0070 \mathrm{a}$ \\
$\mathrm{T} 7$ & $4.315,27 \mathrm{e}$ & $441,00 \mathrm{c}$ \\
\hline C.V. & $\mathbf{7 , 8 2}$ & $\mathbf{1 7 , 2 2}$ \\
\hline
\end{tabular}

*Los datos corresponden al promedio de 4 repeticiones, ${ }^{* *} \mathrm{MH}_{\mathrm{r}}=$ Meloidogyne (Huevos) $/ 100 \mathrm{~g}$ raíz, ${ }^{* * *} 2_{\mathrm{s}}=$ Meloidogyne (J2)/100g suelo; letras distintas indican diferencias significativas.

Conteo de J2 de Meloidogyne spp. La efectividad del aceite esencial en los tratamientos T2 a T5 redujo el número de juveniles de segundo estadio con un promedio aproximado de 281,82 J2/100g al momento de la evaluación final (Tabla 4), teniendo en cuenta que la población inicialmente inoculada del nematodo fue de $\pm 400 \mathrm{~J} 2 / 100 \mathrm{~g}$ suelo, se considera que esta fracción esencial afecta directamente la población inicial y controla la tendencia poblacional a nivel de invernadero por 110 días, duración del ensayo. Respecto a esto Adekunle et al. (2007) afirman que la dihidrotagetona y la ocimenona tienen actividad directa sobre juveniles del género Meloidogyne; de igual forma, Chitwood 
(2002) y Stefanazzi et al. (2006) concluyeron que el efecto depresor de Tagetes sp. sobre las poblaciones de nematodos se debe a la presencia de politienilos y monoterpenos aromáticos afectando los J2.

Además, el análisis estadístico agrupó los tratamientos T1 la menor dosis evaluada de A.E. y al tratamiento T7 sin aplicación del aceite esencial con 357,7 y 441 J2/100g en su orden. En estudios realizados a nivel in vitro con aceites esenciales y extractos acuosos de cinco especies de Tagetes según Adegbite y Adesiyan (2006) señalan que bajas concentraciones de aceites esenciales y extractos acuosos no presentan efectos consistentes sobre el blanco biológico; lo cual lleva a proponer nuevas concentraciones en futuros ensayos con T. zypaquirensis ya que la planta de lulo no se vio afectada por las concentraciones evaluadas buscando un mayor porcentaje de control del nematodo.

En esta investigación, se pudo determinar que el uso del aceite esencial de T. zypaquirensis puede igualar y en algunos casos superar la acción nematicida de la moléculas carbofuran como se describe en la Tabla 4.

\section{CONCLUSIONES}

Mediante cromatografía de gas vinculado a espectrometría de masas (CG-MS) se identificaron tres compuestos principales mayoritarios en el aceite esencial de Tagetes zipaquirensis: dihidrotagetona, E-tagetona y Trans-ocimenona.

La aplicación de aceite esencial de T. zipaquirensis disminuye la severidad de Meloidogyne spp. sobre las variables fitométricas: altura de planta, peso fresco aéreo, peso seco aéreo y peso fresco radical en plántulas de lulo (Solanum quitoense) a nivel de invernadero.

El aceite esencial de T. zypaquirensis presentó actividad biocida sobre las poblaciones del nematodo Meloidogyne spp., afectando el número de huevos del nematodo y número juveniles estadio 2 (J2).

\section{AGRADECIMIENTOS}

Los autores agradecen al Departamento Administrativo de Ciencia, Tecnología e Innovación COLCIENCIAS y a la Vicerrectoría de Investigaciones, Postgrados y Relaciones Internacionales de la Universidad de Nariño por la financiación de esta investigación.

\section{REFERENCIAS BIBLIOGRÁFICAS}

ADEGBITE, A.; ADESIYAN, 0. 2006. Root extracts of plants to control root knot nematode on edible Soybean (Peer reviewed papers). Journal of Vegetable Science. 12(2):5 - 12.

ADEKUNLE, O.; ACHARYA, R.; SINGH, B. 2007. Toxicity of pure compounds isolated from Tagetes minuta oil to Meloidogyne incognita. Australasian Plant Disease. 2:101 - 104 .

ALVAREZ, D.; CASANOVA, L.; CÓRDOBA, K.; OSORIO, 0. 2016. Evaluación de postcosecha y calidad fisicoquímica de genotipos de lulo (Solanum quitoense Lam.) tolerantes a Meloidogyne spp. Vitae. 23(1):785.

BARBOSA, F.; BARBOSA, L.; MELO, E.; BOTELHO, F.; SANTOS, R. 2006. Influência da temperatura do ar de secagem sobre o teor e a composição química do óleo essencial de Lippia alba (Mill). Química Nova. 15 (1):1 - 5.

CASTRO, L.; FLORES, L.; URIBE, L. 2011. Efecto del vermicompost y quitina sobre el control de Meloidogyne sp. en tomate a nivel de invernadero. Agronomía Costarricense. 35(2):21 - 32.

CAVENESS, F.; JENSEN, J. 1955. Modification of the centrifugal flotation technique for the isolation and concentration of nematodes and their eggs from soil and plant tissue. Journal of the Helminthological Society of Washington. 22:87 - 89.

CHAVES, J.; GAVIRIA, F.; TORRES, F.; OBANDO, L. 2002. Análisis de algunos aspectos agroeconómicos del cultivo del lulo (Solanum quitoense L.). Rev. Cienc. Agri. 14(2):178 $-188$.

CHITW00D, J. 2002. Phytochemical based strategies for nematode control. Annual Review of Phytopathology. $40: 221$ - 249 .

DÍAZ, F.; SERRATO, M.; ARCE, M.; LEÓN, J. 2012. Composición del aceite esencial de Tagetes lacera, planta endémica de Baja California del Sur, México. Revista Mexicana de Biodiversidad. 83:543 - 547. 
DI RIENZO, A.; CASANOVES, F.; BALZARINI, M.; GONZÁLEZ, L.; TABLADA, M.; ROBLEDO, W. 2013. InfoStat versión 2013. En: http://www.infostat.com.ar; consulta: agosto, 2015.

EL-SHERIF, G.; EFAEI, R.; NAGAR, E.; SALEM, M. 2007. The role of eggs inoculum level of Meloidogyne sp., on their reproduction and host reaction. African Journal of Agricultural Research. 2(4):159 - 163.

ELYOUSR, A.; KAMAL, A.; ZAKAULLAH, K.; MAGD, E.; MONTASER, F. 2010. Evaluation of plant extracts and pseudomonas spp. For control of root knot nematode, Meloidogyne sp., on tomato. Nematropica. 40(2):12.

ESCOBAR, P.; HERRERA, L.; LEAL1, S.; DURÁN, C.; STASHENKO, E. 2009. Composición química y actividad antitripanosomal de aceites esenciales obtenidos de Tagetes (Fam. Asteraceae), recolectados en Colombia. Revista Salud Universidad Industrial de Santander. 41:280 - 286.

GELPUD, C.; MORA, E.; SALAZAR, C.; BETANCOURTH, C. 2011. Susceptibilidad de genotipos de Solanum spp., al nematodo causante del nudo radical Meloidogyne spp. (chitwood). Acta Agronómica. 60(1):50 - 67.

KIMPINSKI, J.; ARSENAULT, C.; GALLANT, A.; SANDERSON J. 2000. The effect of marigolds (Tagetes sp.) and other cover crops on Pratylenchus penetrans and on following potato crops. Journal of Nematology. 32:531 - 536.

KUMAR, S.; KHANNA, A. 2006. Effect of neem-based products on the root-knot nematode, Meloidogyne sp., and growth of tomato. Nematologia Mediterranea. 34:141 146.

LEHMAN, S. 1979. Factors influencing nematode control with Marigolds. En: http://www.freshfromflorida.com/ content/download/10848/141379/nem050.pdf; consulta: noviembre, 2013.

MURCIA, M.; STASHENKO, E. 2008. Determinación de plaguicidas organofosforados en vegetales producidos en Colombia. Agro Sur. 36(2):11.

MÚNERA, E. 2002. Nematodos asociados con el cultivo del lulo. pp. 135-141. En: Memorias IV Seminario de Frutales de Clima Frío Moderado. Medellín. Colombia.

MADR. MINISTERIO DE AGRICULTURA Y DESARROLLO RURAL. 2012. Anuario estadístico de frutas y hortalizas 2012 y sus calendarios de siembras y cosechas. En: http://207.239.251.112/www/htm3b/public/Anuario/ Anuario_Estadistico2012.pdf; consulta: febrero, 2014.

MADR. MINISTERIO DE AGRICULTURA Y DESARROLLO RURAL. 2013. Anuario estadístico de frutas y hortalizas 2013. En: http://207.239.251.112/www/htm3b/public/ Anuario/ANUARIO_2013.pdf; consulta: febrero, 2015.

OKA, Y.; NACAR, S.; PUTIEVSKY, E.; RAVID, U.; YANIV, Z.; SPIEGEL, Y. 2000. Nematicidal activity of essential oils and their components against the root knot nematode. Phytopathology. 90:710 - 715 .

PARADA, R.; GUZMÁN. F. 1997. Evaluación de extractos botánicos contra el nematodo Meloidogyne sp., en frijol (Phaseolus vulgaris). Agronomía Mesoamericana. 8(1):108 - 114.

PÉREZ, M.; CORTÉS, A.; VILLALOBOS, J.; CASTILLO, P. 2003. Nematicidal activity of essential oils and organic amendments from Asteraceae against root knot nematodes. Plant Pathology. 52:395 - 401.

PLOEG, T. 2000. Effects of amending soil with Tagetes patula $\mathrm{cv}$. single gold on Meloidogyne sp., infestationof tomato. Nematology. 2:289 - 493.

QUINTERO, A.; GONZÁLEZ, N. 2004. Aceite esencial de las hojas Hyptis umbrosa Salzm, extraído por diferentes técnicas. Acta Científica Venezolana. 55:181 - 187.

RODRÍGUEZ, J.; FERNÁNDEZ, J.; REBOLLEDO, L.; URIZA, D. 2001. Uso de extractos vegetales para el combate del nematodo agallador (Meloydogyne Sp.) de la piña (Ananas Comosus (L.) Merr.) en el campo experimental cotaxtla del inifap. p.59. En: Decima Cuarta Reunión Científica-Tecnológica Forestal y Agropecuaria. Veracruz. Mexico.

RIGA, J. 2005. Root extracts of plants to control root knot nematode on edible Soybean (Peer reviewed papers). Journal of Vegetable Science. 12(2):5 - 12.

SERRATO, M.; BARAJAS, J.; DÍAZ, F. 2007. Aceites esenciales del recurso genético Tagetes para el control de insectos, nematos, ácaros y hongos. Agricultura sostenible. 3:181 - 192.

STEFANAZZI, N.; GUTIERREZ, M.; STADLER, T.; BONONI, N.; FERRARI, A. 2006. Actividad biológica del aceite esencial de Tagetes terniflora Kunth (Asteraceae) en Triboleum castaneum Herbst (insecta, coleóptera, tenebrionidae). Boletín de sanidad vegetal de plagas. 32:439 - 447.

TAMAYO, P.; NAVARRO, R.; FORERO, R. 2003. Enfermedades del Lulo en Colombia: Guía de Diagnóstico y Control. Boletín Técnico No 9. Corpoica. 48 p.

TALAMINI, V.; STADNIK, J. 2004. Extractos vegetales e de algas no controle de doenças de plantas. Manejo ecológico de doenças de plantas. 1:45 - 62 .

TAYLOR, A.; SASSER, J. 1983. Biología, identificación y control de nematodos del nudo de la raíz (especies de Meloidogyne). Primera edición. Artes gráficas de la Universidad del estado de Carolina del Norte. 111 p.

TSAY, T; WU, S.; LIN, Y. 2004. Evaluation of Asteraceae plants for control of Meloidogyne sp., Journal of Nematology. 36(1):36 - 41. 